\title{
Optimal Pricing for Service Provisioning in the Secondary Spectrum Market
}

\author{
Fan $\mathrm{Gu}^{1}$, Xianwei $\mathrm{Li}^{2, \mathrm{a}}$, Liang Zhao ${ }^{3}$, Haiyang Zhang ${ }^{4}$, and Xiaoying Yang ${ }^{5}$ \\ ${ }^{1}$ School of Continuing Education, Anhui Institute of International Business, Hefei, China \\ ${ }^{2}$ School of Information Engineering, Suzhou University, Suzhou, China \\ ${ }^{3}$ School of School of Computer Science, Shenyang Aerospace University, Shenyang, China \\ ${ }^{4}$ School of Environment and Geomatics Engineering, Suzhou University, Suzhou, China \\ ${ }^{5}$ School of Information Engineering, Suzhou University, Suzhou, China
}

\begin{abstract}
In this paper, we investigate the problem of spectrum sharing in a secondary spectrum market where one secondary operator provisions network access services to a number of secondary users (SUs) by leasing spectrum from spectrum holder. For the system model under consideration, the spectrum allocated to the secondary operator can be shared by SUs. We model the interaction between the secondary operator and SUs as a two-stage Stackelberg game, where the secondary operator network price decisions in the first stage, and SUs make their spectrum demands decisions in the second stage. We use the backward induction method to solve this game. The numerical results show that the proposed solution method can capture the main factors of the secondary spectrum market, and provide a promising framework for the design of future secondary CR systems.
\end{abstract}

\section{Introduction}

Radio spectrum is recognized as one of the most scare and precious resources in the wireless networks, which is conventionally controlled by government via static license-based allocations. However, according to some recent works, many spectrum bands are not fully utilized even in densely populated urban areas, such Chicago [1][3]. Besides, users' demands for wireless data service have increased rapidly in the past few years. According to a recent report from by Cisco, the monthly global mobile data traffic will reach 49 exabytes by 2021 [4]. The paradox between the rapidly growing demand for wireless data services and low utilization of spectrum allocation suggests that there are some shortcomings for current static spectrum allocation policy.

Cognitive Radio (CR), also known as dynamic spectrum access (DSA), has been proposed as a novel approach to improve the efficiency utilization of spectrum. In Cognitive Radio Networks (CRNs), unlicensed secondary users (SUs) can dynamically access unused part of legacy spectrum bands owned by primary (licensed) users (PUs).

Today, secondary operators, also called mobile virtual network operators (MVNOs), have received significant success worldwide, which is one of the main motivations of our study. MVNOs do not own the physical infrastructure and lease spectrum from spectrum holders to provide services to SUs. For example, IIJmio and LINE MOBILE are two MVNOs in Japan, and Karma is a MVNO in USA.

\footnotetext{
a Corresponding author: lixianwei163@163.com
}

Although a lot of efforts have been devoted to spectrum allocation in CRNs, most of them focus on the technical aspects of spectrum sharing (e.g., designing power control method), in this paper we study from the economic aspect. Moreover, different from previous works that simply analyse homogeneous SUs, which means that all SUs have homogeneous valuation for the spectrum service, we divide SUs into different types based on their preferences for the spectrum quality. For example, some SUs who are watching videos may have higher valuations

The secondary operator sets price for network service to maximize profit

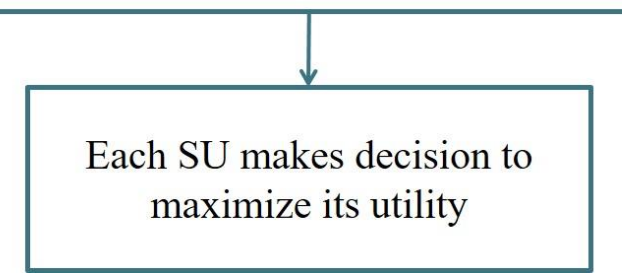

Figure 1. Two-level structure between operators and secondary users. 


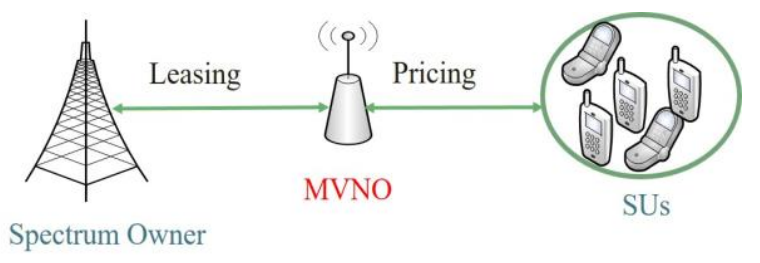

Figure 2. System Model.

of the spectrum while some other SUs who are just phoning have lower valuations on the spectrum [5].

In this paper, we investigate the problem of network services in a secondary spectrum market where a MVNO leases spectrum from the spectrum holder and provides network services to a pool of SUs. The goal of the MVNO is to maximize its own profit. We model the interaction between the MVNO and SUs as a Stakelberg (leader-follower) game, where the MVNO first sets service access prices for the network services to maximize its profits, and then each SU will decide whether to subscribe to network services based on prices and qualities of the services, as illustrated in Fig.1. We use backward induction method to solve the game.

\section{Related Works}

We review and discuss some notable related works that cantered around price-based spectrum service access control in CRNs in this part.

Game theory has been widely used for resource allocation and management in wireless networks in the past few years. Focusing on a duopoly femtocell communications market, Ren et al. studied the problem of long-term entry and spectrum sharing scheme decision from the perspective of an entrant network service provider [9]. Due to users have different preferences for different time slots, Zhang et al. studied time-dependent price competition in a duopoly wireless networks market [10]. But these works did not consider the costs of network service providers.

Spectrum trading and sharing in CRNs have been extensively studied by using game theory. Some works related works analysed the interaction between the primary and secondary operators. The authors in [7] jointly addressed the problem of pricing and network selection in CRNs, where the primary operator who can provide higher guaranteed service and the secondary operator who provides cheaper best-effort secondary network service compete to serve a common pool of users. The problem under consideration is formulated as a a Stackelberg game, where the two operators first set the prices of network services to maximize their revenues. Then, users decide which operator to select. Kinoshita et al. proposed a spectrum sharing method aiming to achieve both users' higher throughput and operators' profit by setting appropriate pricing strategy [11]. However, these previous works have limitations in ignoring users' heterogeneous types or without considering the channel information. Apart from the existing works, we take both users' heterogeneous types and channel information into consideration.

\section{System Model}

In this section, we introduce the system model where one secondary operator, denoted by MVNO, lease spectrum from spectrum owner and provide network service to a number of SUs, as illustrated in Fig.2. The system model that we use is mainly inspired by the works of $\mathrm{Li}$ [8] and Zhao [16] but with different objective functions. As the radio spectrum allocated to spectrum holder remains largely unused even in densely populated urban areas [1], the unused spectrum can form a spectrum pool where the total available bands are divided into a lot of unit channels.

These channels have different qualities due to interference levels, as shown in Fig.3. We assume that SUs purchase network service from the MVNO and have their own preferences for channel quality.

We assume that the channels with quality $q$ are leased to MVNO. The channel quality $q$ can be expressed as [8] [16]

$$
q=B \log _{2}\left(1+\frac{\rho}{I_{\mathrm{i}}}\right)
$$

where $B$ is bandwidth, $\rho$ is the power received by the $\mathrm{SU}$, and $I_{i}$ is the interference of the channel.

\subsection{SUs' Model}

In order to capture SUs' heterogeneous valuations of the spectrum service, we divide SUs into different types based on their different preferences for the spectrum quality. We assume that SUs have different types and the type of SU $k$ is characterized by a parameter $\theta_{k}$ which reflects SU's preference for spectrum quality, and a higher value of $\theta_{k}$ means this user has a higher preference for spectrum quality. For the $\theta_{k}$ SU, its utility function can be expressed as [17] [8] [16]

$$
U_{k}=\theta_{k} q-\pi(q)
$$

where $q$ denotes the spectrum quality of this MVNO, and $\pi(q)$ is the channel price.

\subsection{Secondary Operator's Model}

From the perspective of the secondary operator MVNO, its profit function is denoted as

$$
R_{k}=\pi(q)-C(q)
$$


where $C(q)$ is the channel cost with quality $q$, which is denoted as [18]

$$
C(q)=C_{0}+T(q)=C_{0}+a q^{b}
$$

where, $C_{0}$ is the fixed cost which includes the infrastructure cost, and $a$ and $b$ reflect the impact of channel quality on operating costs and users' sensitivity to price, respectively.

The objective of the MVNO is to maximize its profit. It is obvious that this MVNO would like to sell network service if and only if $\pi(q) \geq C(q)$.

For convenience of analysis, the notations used throughout this paper are summarized in Table 1.

\section{Single Pricing Scheme under Incomplete Information}

In this section, we analyse a secondary spectrum market where one secondary operator sets single price of its service to maximize profit. We consider incomplete information case in this section, that is the secondary operator MVNO does not know the type of each SU, but knows the distribution of user types. We assume that the types of SUs is uniformly distributed [0, 1] with probability distribution function (PDF) $f(\cdot)$ and cumulative distribution function (CDF) $F(\cdot)$. The relationship between the secondary operator and SUs is modelled as a two-stage Stakelberg game, which can be solved by employing the backward induction method [14], [15]. We first analyse the utility maximization of SUs in Stage II based on the price of network service. Then, we investigate how the secondary operator sets network service prices in Stage I.

Table 1. Notations Summary.

\begin{tabular}{|c|c|}
\hline Notation & Description \\
\hline$k$ & subscript of a SU \\
\hline$\pi$ & single price of network service \\
\hline$\pi_{k}$ & price of network service for SU $k$ \\
\hline$R_{k}$ & $\begin{array}{l}\text { the profit of MVNO for selling } \\
\text { network service to user } k\end{array}$ \\
\hline$R_{i}^{*}$ & $\begin{array}{l}\text { optimal profit of MVNO in } \\
\text { incomplete information case }\end{array}$ \\
\hline$R_{c}^{*}$ & $\begin{array}{l}\text { optimal profit of MVNO in } \\
\text { complete information case }\end{array}$ \\
\hline$q$ & spectrum capacity of the MVNO \\
\hline$\theta_{k}$ & $\begin{array}{l}\text { SU k's preference for spectrum } \\
\text { quality }\end{array}$ \\
\hline$f(\cdot)$ & $\begin{array}{l}\text { probability density function (PDF) } \\
\text { of SUs' preferences parameter }\end{array}$ \\
\hline$F(\cdot)$ & $\begin{array}{l}\text { cumulative density function (CDF) } \\
\text { of SUs' preferences parameter }\end{array}$ \\
\hline
\end{tabular}

\begin{tabular}{|c|c|}
\hline$\Omega$ & $\begin{array}{c}\text { the number of users that subscribe } \\
\text { to network services in incomplete } \\
\text { information case }\end{array}$ \\
\hline$\theta^{*}$ & $\begin{array}{c}\text { the marginal point where SUs } \\
\text { switch from negative utility to } \\
\text { positive in incomplete information } \\
\text { case }\end{array}$ \\
\hline$U_{k}$ & $\begin{array}{c}\text { the utility that type } \theta_{k} \text { SU gets from } \\
\text { MVNO }\end{array}$ \\
\hline
\end{tabular}

\subsection{User's Utility Maximization Problem in Stage} II

For the $\mathrm{SU} \theta_{k}$, it will subscribe to network services if and only if

$$
U_{k}=\theta_{k} q-\pi(q) \geq 0
$$

From which, we know that only the types of SUs whose values meet the following requirement subscribe to network services:

$$
\theta_{k} \geq \frac{\pi(q)}{q}
$$

Denote

$$
\theta_{k}^{*}=\frac{\pi(q)}{q}
$$

The number of users that subscribe to network services can be expressed as

$$
\Omega=1-F\left(\theta_{k}^{*}\right)=1-\frac{\pi(q)}{q}
$$

\subsection{MVNO's Profit Maximization Problem in Stage I}

From the perspective of the MVNO, its objective is to maximize profit, which is the difference between its revenue and cost,

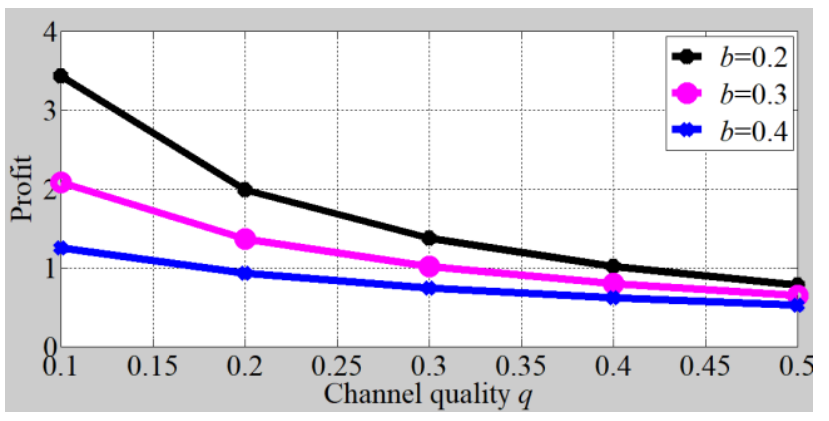

Figure 4. The optimal profit of MVNO versus its channel quality.

$$
\begin{aligned}
R & =\int_{\theta^{*}}^{1}[\pi(q)-C(q)] f(\theta) d(\theta) \\
& =\left(1-\frac{\pi(q)}{q}\right)\left[\pi(q)-a q^{b}\right]
\end{aligned}
$$


It is obvious that the above objective function is a concave function, therefore, by taking the derivative of $\mathrm{R}$ with respective to $\pi$, and setting the equality to zero, we have

$$
\frac{\partial R}{\partial \pi(q)}=\frac{C_{0}+a q^{b}-2 \pi(q)+q}{q}=0
$$

From which we get

$$
\pi(q)=\frac{C_{0}+a q^{b}+q}{2}
$$

By substituting Eq.(11) in to Eq.(9), we get the optimal profit of the MVNO,

$$
R^{*}=\pi(q)=\frac{\left[q-\left(C_{0}+a q^{b}\right)\right]^{2}}{4 q}
$$

\section{Numerical Results}

In this section, we present numerical results to validate the performance of our analysis. Based on [18], the default parameters are set as follows unless otherwise stated: $C_{0}=0.01, q=0.4, a=2$ and $b=3$.

Fig. 4 shows how the optimal profit of MVNO varies with its channel quality in the incomplete information case. This figure suggests that the profit of the MVNO firstly decreases with channel quality increasing due to the reason that the MVNO sets single price for the SUs in the incomplete information case. This figure also shows that if cost parameter $b$ increases, the profit of the MVNO decreases, which means that as the increment of cost for a unit quality increase, the MVNO will get less profit.

\section{Conclusions}

In this paper, we studied price differentiation in a secondary spectrum market, where the idle spectrums with different qualities are leased to a secondary operator who provide service access to SUs with the objective of maximizing its profits. Our numerical results show that the MVNO can improve the channel quality to improve its profits in the two information cases, and the MVNO gets less profit if its operating costs increases. In the incomplete information case, the profit of the MVNO first increases as the channel quality increases, then decreases with the channel quality increasing.

\section{Acknowledgement}

This work is partly supported by Natural science research project of University of Anhui Province (KJ2016A125), the National Science Foundation for Young Scientists of China (61701322), the Key Projects of Liaoning Natural Science Foundation (20170540700), the Liaoning Provincial Department of Education Science Foundation (L201630), the project of College Students Construction Plan of Anhui Province (S2015ckjh150, S2016ckjh053), the Major Project of Natural Science of Education Department of Anhui Province (KJ2014ZD31), Key Scientific Research Projects of Suzhou University (2016yzd10, 2015ykf13), Key projects of the youth talent support program of Suzhou University (2016YQNRC004), and Suzhou Regional Collaborative Innovation Center (2016szxt05, 2016szxt06).

\section{References}

1. L. Duan, J. Huang, and B. Shou, Duopoly competition in dynamic spectrum leasing and pricing, IEEE T. Mobile Comput., vol.11, no.11, pp.1706-1719, Nov.2012.

2. L. Duan, J. Huang, and B. Shou, Investment and pricing with spectrum uncertainty: A cognitive operators perspective, IEEE T. Mobile Comput., vol.10, no.11, pp.1590-1604, Nov.2011.

3. M. S.Khan, M. Usman, and V.V.Hiep et al., Efficient selection of users pair in cognitive radio network to maximize throughput using simultaneous transmit-sense approach, IEICE Trans. Commmu., vol.E100-B, no.2, pp.380-389, Feb.

4. Ciscovisualnetworkingindex:Forecastandmethodol ogy, 2016-2021. https://www.cisco.com/c/en/us/solutions/collateral / service-provider/visual-networking-indexvni/mobile-white-paper-c11-520862.html. [ accessed on Auguset 10, 2017].

5. X. Cao, Y. Chen, and K.J.R. Liu, Cognitive radio networks with heterogeneous users: How to procure and price the spectrum?, IEEE T. Commun., vol.14, no.3, pp.1676-1688, March 2015.

6. D. Niyato and E. Hossain, A game theoretic analysis of service competition and pricing in heterogeneous wireless access networks, IEEE $\mathrm{T}$. Commun., vol.7, no.12, pp.5150-5155, Dec.2008.

7. J. Elias, F. Martignon, and L. Chen et al., Joint operator pricing and network selection game in cognitive radio networks: Equilibrium, system dynamics and price of anarchy, IEEE T. Veh Technol., vol.62, no.9, pp.4576-4589, Nov.2013.

8. F. Li, Z. Sheng, and J. Hua et al, Preference-based spectrum pricing in dynamic spectrum access networks, IEEE T. Serv. Comput., in press.

9. S.Ren, K.Park, andM.Schaar, Entry and spectrum sharings cheme selection in femtocell 
communications markets, IEEE/ACM Trans. Netw., vol.21, no.1, pp.218-232, Feb. 2013.

10. C. Zhang, B. Gu, and K. Yamori et al., Duopoly competition in time-dependent pricing for improving revenue of network service providers, IEICE Trans. Commmu., vol.E96-B, no.12, pp.2964-2975, Dec. 2015.

11. K.Kinoshita, Y.Maruyama, and K.Kawano et al., A spectrum sharing method based on users behavior and providers profit, IEICE Trans. Commmu., vol.E100-B, no.10, pp.1928-1938, Nov. 2017.

12. N.H. Tran, C.S. Hong, and Z. Han et al., Optimal pricing effect on equilibrium behaviors of delaysensitive users in cognitive radio networks, IEEE Trans. J. Sel. Areas Commun., vol.31, no.11, pp.2566-2579, Nov. 2013.

13. N.H. Tran, L.B. Le, S. Ren, Z. Han, and C.S. Hong, Joint pricing and load balancing for cognitive spectrum access: Non-cooperation versus cooperation, IEEE Trans. J. Sel. Areas Commun., vol.33, no.5, pp.972-985, May 2015.

14. D. Fudenberg and J. Tirole, Game theory, MIT Press, Cambridge, MA, USA, 1991.

15. Z. Han, D. Niyato, and W. Saad et al., Game theory in wireless and communication networks: Theory, models, and applications, Cambridge University Press, Cambridge, UK, 2011.

16. S. Zhao, Q. Zhu, and G. Zhu et al., Competitions and dynamics of mvnos in spectrum sharing: An evolutionary game approach, IEICE Trans. Commmu., vol.E96-B, no.1, pp.69-72, Jan.2013.

17. X.J. Tan, L. Li, and W. Guo, A game-theoretic approach for opportunistic spectrum sharing in cognitive radio networks with incomplete information, IEICE Trans. Commmu., vol.95, no.4, pp.1117-1124, April 2012.

18. L. Gao, X. Wang, Y. Xu, and Q. Zhang, Spectrum trading in cognitive radio networks: A contracttheoretic modeling approach, IEEE J. Sel. Areas Commun., vol. 29, no. 4, pp. 843-855, April 2011. 